\title{
Uso de chupeta em pré-escolares do sul do Brasil: prevalência e fatores associados
}

\author{
Pacifier use in preschoolers from southern Brazil: \\ prevalence and associated factors
}

\author{
Fabiana Vargas-Ferreira* \\ Rita de Azevedo Senna** \\ Mariana Cezar Ilha*** \\ Patrícia Figueiró ${ }^{* * * *}$ \\ Carlos Alberto Feldens ${ }^{* * * *}$ \\ Paulo Floriani Kramer
}

\section{Resumo}

Objetivo: investigar a prevalência e os fatores associados ao uso de chupeta em pré-escolares. Sujeitos e método: estudo transversal foi realizado com 1.316 crianças de 0 até 5 anos de idade no município de $\mathrm{Ca}$ noas, no Rio Grande do Sul. O instrumento de pesquisa foi um questionário semiestruturado respondido pelos responsáveis sobre características sociodemográficas (sexo, cor da pele, idade da criança e materna, escolaridade materna, estrutura e renda familiar) e comportamentais (uso da mamadeira e aleitamento materno). O desfecho foi presença/ausência de uso de chupeta. A análise estatística compreendeu regressão de Poisson com variância robusta bruta e ajustada. Resultados: a prevalência de uso de chupeta em algum momento da vida foi de $68,1 \%$. A análise multivariável ajustada mostrou que crianças que usaram mamadeira tiveram três vezes maior prevalência do desfecho (RP: 3,21; IC95\% 2,25-4,59; $p<0,001$ ) se comparadas àquelas que nunca usaram mamadeira. Além disso, a análise adicional encontrou que crianças mais novas ainda usam a chupeta quando comparadas às mais velhas. Conclusão: conclui-se que a prevalência do uso de chupeta é alta e está associada com hábitos de nutrição. Assim, entender a associação de aspectos biopsicossociais e sua rede de causalidade torna-se essencial para o estabelecimento de estratégias de promoção de saúde na primeira infância.

Palavras-chave: Chupeta. Criança. Saúde pública.

\section{Introdução}

Hábitos de sucção não nutritiva (HSNN) são comuns na infância e podem determinar impacto negativo no crescimento e desenvolvimento craniofacial ${ }^{1-3}$. Desde a vida intrauterina, o bebê apresenta o reflexo natural de sucção não nutritiva e, ao nascimento, a função está completamente desenvolvida $^{4}$. Esse tipo de sucção supre carências psicológicas e afetivas, promovendo sensações de bem-estar, prazer emocional, conforto e proteção ${ }^{5}$. $\mathrm{O}$ uso da chupeta é o mais prevalente dos HSNN, sendo um dispositivo amplamente utilizado por crianças em todo o mundo ${ }^{6}$. Estudos de base populacional têm demonstrado prevalências que variam de $25 \%$ a $78 \%{ }^{6-10}$. Essas diferenças normalmente são explicadas por questões culturais, socioeconômicas e comportamentais, embora a literatura ainda apresente resultados contraditórios e explicações insatisfatórias.

Uma das maiores preocupações dos profissionais da área da saúde refere-se à influência dos HSNN sobre aleitamento materno e maloclusão. A literatura tem enfatizado que é preciso evitar a exposição da criança à chupeta até o aleitamento materno estar completamente estabelecido, por volta de três até quatro semanas após o nascimento ${ }^{11}$. Essa orientação permite que se evite o fenômeno de "confusão de bicos", uma vez que o uso concomitante de chupeta e mamadeira potencializa o risco para a

\footnotetext{
Doutora em Epidemiologia. Professora adjunta, Departamento de Odontologia Social e Preventiva, Universidade Federal de Minas Gerais, Belo Horizonte, Minas Gerais, Brasil.

Mestre em Odontologia, Departamento de Odontopediatria, Universidade Luterana do Brasil, Canoas, Rio Grande do Sul, Brasil.

Mestre em Odontologia, Departamento de Odontopediatria, Universidade Luterana do Brasil, Canoas, Rio Grande do Sul, Brasil.

Cirurgiã-dentista especialista em Odontopediatria, Universidade Luterana do Brasil, Canoas, Rio Grande do Sul, Brasil.

***** Doutor em Epidemiologia. Professor adjunto, Departamento de Odontopediatria, Unniversidade Luterana do Brasil, Canoas, Rio Grande do Sul, Brasil.

${ }^{*}$ Doutor em Odontologia. Professor adjunto, Departamento de Odontopediatria, Universidade Luterana do Brasil, Canoas, Rio Grande do Sul, Brasil.
} 
cessação do aleitamento materno no primeiro ano de vida ${ }^{11-12}$. Além disso, evidências sugerem que a sucção sobre a região da auréola da mama determina uma postura apropriada para respirar pelas fossas nasais, com a língua na posição adequada, favorecendo o desenvolvimento do sistema estomatognático $^{13}$. O uso da chupeta, por sua vez, mantém a língua em posição mais baixa impedindo aplicação da pressão necessária contra o palato para o crescimento transversal maxilar ${ }^{14}$. Dessa forma, a presença dos HSNN tem sido associada com maloclusão, principalmente mordida aberta anterior e mordida cruzada posterior ${ }^{2}$.

Orientações e estratégias de promoção de saúde que envolvam hábitos de sucção nutritiva e não nutritiva dependem da compreensão de sua rede de causalidade e fatores associados. São escassos, contudo, os estudos epidemiológicos de base populacional que avaliam fatores sociodemográficos e comportamentais e que empregam análise multivariável para controle de confusão $0^{9,10,15}$. Assim, o objetivo do presente estudo foi estimar a prevalência e os fatores associados ao uso da chupeta em pré-escolares do sul do Brasil.

\section{Sujeitos e método}

\section{Considerações éticas}

Este estudo obteve aprovação do Comitê de Ética e Pesquisa da Universidade Luterana do Brasil (Ulbra) e foi conduzido de acordo com a Resolução 196/96 (protocolo número 2010-056H). Além disso, os responsáveis assinaram o termo de consentimento livre e esclarecido, concordando com a participação das crianças.

\section{Participantes e delineamento}

Este estudo transversal foi conduzido com pré-escolares com idade de 0 a 5 anos da cidade de Canoas, no sul do Brasil. A cidade tem, aproximadamente, 324.000 habitantes, e todas as casas têm acesso à água de abastecimento público com flúor (nível de 0,8 ppm). A população do estudo compreendeu 1.732 crianças, assistidas por 31 escolas públicas, e seus responsáveis.

\section{Coleta dos dados}

O trabalho de campo envolveu seis equipes formadas por dentistas e auxiliares (acadêmicos de Odontologia). Os dados foram coletados por meio de entrevista padronizada com os responsáveis. O treinamento foi realizado com pesquisadores com experiência prévia em levantamentos epidemiológicos.

Um questionário foi elaborado para obter informações sobre variáveis demográficas: sexo da criança (masculino/feminino), cor da pele (coletada conforme as categorias do Instituto Brasileiro de Geografia e Estatística e categorizada em branca e não branca), idade da criança (coletada em anos e categorizada em 0-2 e 3-5 anos), idade materna ao nascimento da criança (coletada em anos e categorizada em <20, 21-35, >35); socioeconômicas: renda familiar (coletada na moeda brasileira - reais e categorizada em tercis), estrutura familiar (nuclear e não nuclear) e escolaridade materna (anotada em anos de estudo e categorizada em $\leq 8,9-11,>11$ ). As variáveis comportamentais coletadas foram referentes a aleitamento materno, uso de mamadeira e uso de chupeta. $\mathrm{O}$ aleitamento materno foi coletado em meses de duração total e categorizado em: sim ( $\geq 1$ mês) e não ( $<1$ mês ou sem aleitamento materno). Os usos de mamadeira e chupeta foram coletados como: "nunca usou", "usou e não usa mais" e "ainda usa", sendo categorizados em: não (nunca usou) e sim (ainda usa ou usou e não usa mais).

\section{Análise de dados}

Para análise estatística, foi utilizado o software Stata versão 12.0 (Stata Corp. College Station, TX, USA). Análises descritiva e bivariada foram conduzidas para avaliar a associação entre preditores e o desfecho uso de chupeta, utilizando-se os testes Qui-Quadrado e de Tendência Linear $(\mathrm{p}<0,05)$. Para a análise multivariável, utilizou-se a regressão de Poisson com variância robusta (razão de prevalência - RP com intervalo de confiança de 95\%). O modelo hierárquico foi composto por três níveis: distal - demográfico (sexo, idade da criança e materna, cor da pele); intermediário - socioeconômico (renda familiar, estrutura familiar e escolaridade materna); e proximal - comportamental (uso de mamadeira e aleitamento materno). $\mathrm{O}$ procedimento adotado foi backward stepwise, para selecionar as variáveis em cada nível. O modelo final estimou as RP ajustadas para variáveis do mesmo nível e acima. Variáveis preditoras com $\mathrm{p}<0,05$ foram mantidas no modelo de regressão final.

\section{Resulltados}

Das 1.732 crianças com idade de 0 a 5 anos assistidas em pré-escolas públicas, 1.316 fizeram parte do estudo $(76 \%)$. As razões para as perdas foram: ausência de participação no dia do levantamento, ausência de termo de consentimento assinado pelos responsáveis e recusa em participar.

A maioria da amostra foi composta por crianças do sexo masculino (52\%), com média de idade de 3,2 anos $(\mathrm{DP}+/-1,3)$ e de cor branca $(67 \%)$; a escolaridade materna variou de 0 até 18 anos, com média de 9,5 anos (DP 2,7), sendo que 54,4\% das mães tinham entre 9 e 11 anos de estudo; a maioria das crianças era de família nuclear $(65,2 \%)$; a idade materna mais prevalente foi na faixa de 20-35 anos 
(79\%). Já a prevalência do aleitamento materno foi de $78 \%$ e do uso da mamadeira, de $91 \%$.

A prevalência de uso de chupeta em alguma fase da vida foi de 68,1\% (IC95\% 65,5\%-70,6\%), sendo que $49,3 \%$ das crianças usavam chupeta "atualmente". Na análise bivariada, crianças do sexo feminino $(\mathrm{p}=0,032)$, com idade de 0 a 2 anos $(\mathrm{p}=0,046)$ e que faziam uso de mamadeira $(\mathrm{p}<0,001)$ tiveram maior prevalência de uso de chupeta (Tabela 1 ).

Tabela 1 - Características da amostra, prevalência de uso de chupeta e variáveis independentes, Canoas, RS, Brasil, 2010

\begin{tabular}{|c|c|c|c|c|c|}
\hline \multirow{2}{*}{ Variáveis } & \multirow{2}{*}{$\mathrm{N}$} & \multirow{2}{*}{$(\%)$} & \multicolumn{2}{|c|}{ Uso de chupeta } & \multirow{2}{*}{$\mathrm{p}^{*}$} \\
\hline & & & $\mathrm{N}$ & $(\%)$ & \\
\hline \multicolumn{5}{|l|}{ Sexo } & $0,032 *$ \\
\hline Masculino & 684 & $(52,0)$ & 446 & $(65,5)$ & \\
\hline Feminino & 632 & $(48,0)$ & 448 & $(71,0)$ & \\
\hline \multicolumn{5}{|l|}{ Idade da criança } & $0,046^{*}$ \\
\hline $0-2$ anos & 439 & $(33,4)$ & 315 & $(71,5)$ & \\
\hline 3-5 anos & 877 & $(66,7)$ & 579 & $(66,3)$ & \\
\hline \multicolumn{5}{|l|}{ Cor da pele } & $0,471 *$ \\
\hline Branco & 880 & $(67,0)$ & 604 & $(68,8)$ & \\
\hline Não branco & 436 & $(33,0)$ & 290 & $(66,8)$ & \\
\hline \multicolumn{5}{|l|}{ Idade materna } & $0,498^{* *}$ \\
\hline$<20$ anos & 117 & $(9,0)$ & 75 & $(64,1)$ & \\
\hline 20-35 anos & 1.020 & $(79,0)$ & 707 & $(69,5)$ & \\
\hline$>35$ anos & 155 & $(12,0)$ & 95 & $(61,7)$ & \\
\hline \multicolumn{5}{|c|}{ Escolaridade materna } & $0,810^{* *}$ \\
\hline$\leq 8$ anos & 463 & $(36,2)$ & 478 & $(68,7)$ & \\
\hline 9-11 anos & 698 & $(54,4)$ & 307 & $(66,3)$ & \\
\hline$>11$ anos & 121 & $(9,4)$ & 84 & $(70,0)$ & \\
\hline \multicolumn{5}{|l|}{ Estrutura familiar } & $0,365^{*}$ \\
\hline Nuclear & 858 & $(65,2)$ & 576 & $(67,3)$ & \\
\hline Não nuclear & 458 & $(34,8)$ & 318 & $(69,7)$ & \\
\hline \multicolumn{5}{|c|}{ Renda familiar (tercis) } & $0,397^{* *}$ \\
\hline 1 (menor) & 423 & $(34,5)$ & 281 & $(66,4)$ & \\
\hline 2 & 372 & $(30,3)$ & 252 & $(67,9)$ & \\
\hline 3 (maior) & 432 & $(35,2)$ & 298 & $(69,1)$ & \\
\hline \multicolumn{5}{|c|}{ Aleitamento materno } & $0,682 *$ \\
\hline Não & 279 & $(22,0)$ & 191 & $(68,7)$ & \\
\hline Sim & 991 & $(78,0)$ & 668 & $(67,4)$ & \\
\hline \multicolumn{5}{|c|}{ Uso de mamadeira } & $<0,001^{*}$ \\
\hline Não & 108 & $(8,3)$ & 24 & $(22,2)$ & \\
\hline Sim & 1.197 & $(91,0)$ & 865 & $(70,5)$ & \\
\hline
\end{tabular}

* Teste Qui-Quadrado $(p<0,05)$.

** Teste Qui-Quadrado de tendência linear $(p<0,05)$.

Fonte: autores.

Após ajuste para potenciais confundidores (Tabela 2), crianças do sexo feminino apresentaram uma prevalência 8\% maior de uso de chupeta quando comparadas às crianças do sexo masculino (RP 1,08; IC95\% 1,01-1,6), sendo que as crianças de menor idade apresentaram $7 \%$ maior probabilidade de usar chupeta quando comparadas às de maior idade (RP 1,07; IC95\% 1,01-1,16). Crianças que usavam ou haviam usado mamadeira apresentaram uma probabilidade três vezes maior de uso de chupeta (RP 3,21; IC95\% 2,25-4,59).
Tabela 2 - Razões de prevalência brutas e ajustadas $(R P)$ e intervalos de confiança 95\% (IC 95\%) da ocorrência de uso de chupeta de acordo com as variáveis independentes, Canoas, RS, Brasil, 2010

\begin{tabular}{l|c|c|c|c|c|c}
\hline \multirow{2}{*}{ Variáveis } & \multicolumn{3}{|c|}{ Modelo bruto } & \multicolumn{3}{c}{ Modelo multivariável* $^{*}$} \\
\cline { 2 - 7 } & RP & IC $95 \%$ & p & RP & IC 95\% & P \\
\hline
\end{tabular}

Nível 1 - Demográficas

\begin{tabular}{l|l|l|l|l|l|l}
\hline $\begin{array}{c}\text { Sexo } \\
\text { Feminino }\end{array}$ & 1,08 & $(1,01-1,17)$ & & 1,08 & $(1,01-1,16)^{\#}$ & 0,047 \\
$\begin{array}{c}\text { Masculino } \\
\text { Cor da pele } \\
\text { Branco }\end{array}$ & 1,00 & & & 1,00 & & \\
$\quad \begin{array}{l}\text { Não branco } \\
\text { Idade da criança }\end{array}$ & 1,03 & $(0,95-1,11)$ & & & & \\
$\quad$-2 anos & 1,08 & $(1,01-1,17)$ & & 1,07 & $(1,01-1,16)^{\#}$ & \\
3-5 anos & 1,00 & & & 1,00 & & \\
Idade materna & & & 0,516 & & & \\
$<20$ anos & 1,04 & $(0,86-1,25)$ & & & & \\
$20-35$ anos & 1,13 & $(0,98-1,28)$ & & & & \\
$>35$ anos & 1,00 & & & & & \\
\hline
\end{tabular}

Nível 2 - Socioeconômicas

\begin{tabular}{l|l|l|l|l|l|l}
\hline $\begin{array}{l}\text { Escolaridade paterna } \\
\leq 8 \text { anos }\end{array}$ & 0,95 & $(0,83-1,08)$ & & & & \\
$9-11$ anos & 0,98 & $(0,86-1,11)$ & & & & \\
$>11$ anos & 1,00 & & & & & \\
$\begin{array}{l}\text { Estrutura familiar } \\
\text { Não nuclear }\end{array}$ & 1,04 & $(0,96-1,12)$ & & & & \\
$\quad$ Nuclear & 1,00 & & & & & \\
Renda familiar (tercis) & & & 0,397 & & $\#$ & \\
1 (menor) & 0,96 & $(0,87-1,05)$ & & & & \\
2 & 0,98 & $(0,89-1,08)$ & & & & \\
3 (maior) & 1,00 & & & & & \\
\hline
\end{tabular}

Nível 3 - Comportamentais

\begin{tabular}{l|l|l|l|l|c|c}
\hline $\begin{array}{l}\text { Aleitamento materno } \\
\text { Não }\end{array}$ & 1,02 & $(0,93-1,11)$ & & & & \\
Sim & 1,00 & & & & & \\
Uso de mamadeira & & & $<0,001$ & & & $<0,001$ \\
Sim & 3,24 & $(2,26-4,61)$ & & 3,21 & $(2,25-4,59)$ & \\
Não & 1,00 & & & 1,00 & & \\
\hline
\end{tabular}

\# Variáveis não incluídas ou não permaneceram no modelo multivariável.

Fonte: autores.

A Tabela 3 demonstra a redução significativa no hábito de usar a chupeta "atualmente", à medida que aumenta a idade da criança, de $68,1 \%$ em crianças menores de 2 anos a $32,8 \%$ em crianças de 4 a 5 anos.

Tabela 3 - Associação entre a idade e uso de chupeta (nunca usou e/ ou não usa mais $x$ ainda usa) em pré-escolares, Canoas, RS, Brasil, 2010

\begin{tabular}{|c|c|c|c|c|c|c|}
\hline \multirow{3}{*}{ Variáveis } & \multicolumn{5}{|c|}{ Chupeta } & \multirow{3}{*}{$\mathrm{p}^{*}$} \\
\hline & \multicolumn{3}{|c|}{ Ainda usa } & \multicolumn{2}{|c|}{$\begin{array}{c}\text { Nunca usou e/ou } \\
\text { não usa mais }\end{array}$} & \\
\hline & $\mathrm{N}$ & $\mathrm{N}$ & $\%$ & $\mathrm{~N}$ & $\%$ & \\
\hline Idade da criança & & & & & & $<0,001$ \\
\hline$<2$ anos & 204 & 139 & 68,1 & 65 & 32,0 & \\
\hline 2-3 anos & 550 & 325 & 59,0 & 225 & 41,0 & \\
\hline$>3$ anos & 558 & 183 & 32,8 & 375 & 67,2 & \\
\hline
\end{tabular}

*Teste Qui-Quadrado de tendência linear $(p<0,05)$.

Fonte: autores. 


\section{Discussão}

O principal resultado do presente estudo foi a identificação da alta prevalência de uso de chupeta em pré-escolares no sul do Brasil e sua forte associação com o uso de mamadeira. Compreender os aspectos biopsicossociais associados aos hábitos de sucção nutritiva e não nutritiva, assim como a sua rede de causalidade para diferentes agravos, é a base para o estabelecimento de estratégias de promoção de saúde na primeira infância. A forte associação observada indica que ambos os hábitos devem ser o foco de intervenções para redução de desfechos negativos de saúde infantil.

A maioria dos estudos de base populacional relata proporções superiores a $20 \%$ com relação à chupeta, o que demonstra a magnitude do desfe$\mathrm{cho}^{8,10}$. Tem sido sugerido que características socioeconômicas e comportamentais podem explicar as diferenças ${ }^{16}$. Estudos têm indicado que a industrialização e a modernização da sociedade, associadas à participação das mulheres no mercado de trabalho, poderiam levar à redução do período de aleitamento materno, incentivando assim a oferta de chupeta e mamadeira ${ }^{17,18}$. Além disso, a chupeta pode ser ofertada pelos responsáveis para acalmar o choro das crianças, tendo assim um provável efeito psicológico de segurança e conforto ${ }^{18}$.

$\mathrm{O}$ uso da chupeta em larga escala tem implicações relacionadas à saúde bucal. HSNN prolongados ocasionam a hiperfunção do músculo bucinador, que causa uma deficiência no crescimento transversal ${ }^{8}$. Além disso, um estudo de coorte de nascidos vivos mostrou que o tempo do uso de chupeta foi o mais importante fator de risco para o desenvolvimento da mordida aberta anterior e cruzada posterior aos 6 anos de idade ${ }^{2}$. É importante destacar que a maloclusão em pré-escolares tem impacto na qualidade de vida relacionada à saúde bucal ${ }^{19,20}$.

Neste estudo, indivíduos do sexo feminino apresentaram maior prevalência do uso de chupeta, concordando com os achados de outras investigações $\mathrm{e}^{3,9,21}$, embora alguns estudos evidenciem associação com sexo masculino ${ }^{18,22,23}$. A controvérsia continua e há ausência de justificativa plausível para explicar as diferenças encontradas. Assim, sugerem-se estudos com temática qualitativa para investigar possíveis influências culturais e psicológicas sobre a relação entre sexo e uso de chupeta.

Os achados do estudo mostraram que a maior prevalência do uso de chupeta (usaram e ainda usam) foi entre as crianças mais jovens. Esse resultado pode estar associado ao fato de que as mães apresentaram viés de memória, pois o uso de chupeta é cumulativo, assim, as mães tendem a subestimar a prevalência da chupeta entre as crianças mais velhas. A análise adicional mostrou que as crianças que ainda usam chupeta são as mais jovens, já entre as que nunca usaram e/ou não usam mais, a prevalência foi maior entre as mais velhas. A explicação plausível para esta associação é que, à medida que a idade aumenta, há uma tendência natural de interrupção do hábito, pois as crianças o abandonam por si mesmas ou por sentirem vergonha dos amigos ${ }^{6}$. O resultado é relevante e positivo, uma vez que o dano causado pelos HSNN pode ser corrigido espontaneamente ${ }^{3}$.

No presente estudo, de cada dez crianças, nove relataram o uso da mamadeira, demonstrando que esse hábito é altamente prevalente na infância. Além disso, aquelas que utilizaram mamadeira apresentaram uma probabilidade três vezes maior de usar chupeta. Inúmeros estudos também têm demonstrado que o uso da mamadeira está fortemente associado ao uso de chupeta ${ }^{7,10,21}$. Um estudo longitudinal demonstrou que crianças que usavam mamadeira por período superior a dois anos apresentavam maior incidência do uso de chupeta ${ }^{21}$. Assim, a mamadeira é considerada um fator de risco para uso da chupeta ${ }^{8,24}$.

Dados amostrais representativos do Brasil revelam que o tempo de aleitamento materno exclusivo mediano foi de 1,4 meses e a variação da prevalência de aleitamento materno foi de 72,1\% (Região Sul) até $87,7 \%$ (Região Norte) ${ }^{25}$. Uma revisão sistemática, por sua vez, mostrou que, em países de renda média e baixa, somente $36 \%$ das crianças menores de 6 meses têm aleitamento materno exclusivo ${ }^{26}$. No presente estudo, não foi observada associação entre aleitamento materno e uso de chupeta, o que possivelmente ocorreu em função do ponto de corte utilizado para aleitamento materno. Um estudo de coorte realizado em São Leopoldo, RS, contudo, mostrou que o uso da chupeta em consonância com o uso da mamadeira no primeiro mês de vida da criança impacta negativamente a continuidade do aleitamento materno no primeiro ano de vida, indicando que há necessidade de orientação específica para as mães ${ }^{12}$. Evidências têm sugerido que crianças amamentadas por longos períodos têm menor morbimortalidade, menor ocorrência de maloclusão e maior inteligência ${ }^{26}$. Além disso, essa prática protege contra sobrepeso e diabetes mellitus na vida adulta $^{26}$ e estimula o crescimento anteroposterior da mandíbula ${ }^{21}$.

A literatura ainda é escassa e controversa em relação à influência de fatores socioeconômicos no uso de chupeta ${ }^{9,10,15}$. No presente estudo, não foi observada associação entre as variáveis socioeconômicas e o desfecho, sugerindo que tal influência não ocorra e indicando a necessidade de mais estudos para compreender seu mecanismo.

$\mathrm{O}$ estudo apresenta aspectos importantes a serem considerados. $\mathrm{O}$ tamanho amostral confere poder ao estudo e permite que medidas precisas sejam reportadas. $\mathrm{O}$ uso de análise multivariável para controle de confusão também é um aspecto positivo, assim como a avaliação de variáveis demográficas, socioeconômicas e comportamentais sobre o des- 
fecho. A principal limitação do estudo refere-se ao delineamento transversal e ao viés da causalidade reversa. Apesar dessa possibilidade, a associação entre mamadeira e chupeta preenche alguns requisitos de causalidade propostos por Hill, como força de associação e consistência do achado, minimizando a ocorrência do viés de causalidade reversa. Também há possibilidade de viés de memória, uma vez que as informações tanto das exposições quanto do desfecho são dependentes da memória materna.

\section{Conclusão}

Neste estudo, observou-se uma alta prevalência de utilização de chupeta, indicando a necessidade de promover estratégias educativas para prevenir e reduzir esse hábito. Além disso, a forte associação descrita com uso de mamadeira demonstra que ambos os hábitos devem ser objeto de intervenção, com vistas a promover a saúde infantil.

\section{Abstract}

Objective: to investigate the prevalence and the factors associated with pacifier use by preschoolers. Subjects and method: cross-sectional study performed with 1316 children from zero to five years of age, in the city of Canoas, RS, Brazil. The research instrument was a semi-structured questionnaire answered by the responsible persons on sociodemographic (sex, skin color, child and maternal age, maternal level of education, and family structure and income) and behavioral (bottle-feeding and breastfeeding) characteristics. The outcome was the presence/absence of pacifier use. The statistical analysis included Poisson regression with crude and adjusted robust variance. Results: the prevalence of pacifier use at some point in life was $68.1 \%$. The adjusted multivariate analysis showed that bottle-fed children presented a threefold higher prevalence of the outcome (PR: $3.21,95 \% \mathrm{Cl} 2.25-4.59, \mathrm{p}<0.001)$ compared to those who had never used the bottle. Moreover, an additional analysis found that younger children still use the pacifier compared to older ones. Conclusion: it is concluded that the prevalence of pacifier use is high and it is associated with nutrition habits. Thus, understanding the association of biopsychosocial aspects and their causality network becomes essential for establishing health promotion strategies in early childhood.

Keywords: Pacifier. Child. Public health.

\section{Referências}

1. Karjalainen S, Rönning O, Lapinleimu H, Simell O. Association between early weaning, non-nutritive sucking habits and occlusal anomalies in 3-year-old Finnish children. Int J Paediatr Dent 1999; 9(3):169-73.

2. Peres KG, Barros AJ, Peres MA, Victora CG. Effects of breastfeeding and sucking habits on malocclusion in a birth cohort study. Rev Saúde Pública 2007; 41(3):343-50.
3. Vasconcelos FM, Massoni AC, Heimer MV, Ferreira AM, Katz CR, Rosenblatt A. Non-nutritive sucking habits, anterior open bite and associated factors in Brazilian children aged 30-59 months. Braz Dent J 2011; 22(2):140-5.

4. Sexton S, Natale R. Risks and benefits of pacifiers. Am Fam Physician 2009; 79(8):681-5.

5. Turgeon-O'Brien H, Lachapelle D, Gagnon PF, Larocque I, Maheu-Robert LF. Nutritive and nonnutritive sucking habits: a review. ASDC J Dent Child 1996; 63(5):321-7.

6. Garbin CA, Garbin AJ, Martins RJ, Souza NP, Moimaz SA. Prevalence of non-nutritive sucking habits in preschoolers and parents' perception of its relationship with malocclusions. Ciênc Saúde Coletiva 2014; 19(2):553-8.

7. Vadiakas G, Oulis C, Berdouses E. Profile of non-nutritive sucking habits in relation to nursing behavior in pre-school children. J Clin Pediatr Dent 1998; 22(3):133-6.

8. Degan VV, Puppin-Rontani RM. Prevalence of pacifier-sucking habits and successful methods to eliminate them - a preliminary study. J Dent Child 2004; 71(2):148-51.

9. Santos AS, Holand ALF, Sena MF, Gondim LAM, Ferreira MAF. Nonnutritive sucking habits among preschool-aged children. J Pediatr 2009; 85(5):408-14.

10. Góes MPS, Araújo CMT, Góes PSA, Jamelli SR. Non-nutritive sucking habits persistence: prevalence and associated factors. Rev Bras Saúde Mater Infant 2013; 13(3):247-57.

11. Nelson AM. A comprehensive review of evidence and current recommendations related to pacifier usage. J Pediatr Nurs 2012; 27(6):690-9.

12. Feldens CA, Vitolo MR, Rauber F, Cruz LN, Hilgert JB. Risk factors for discontinuing breastfeeding in southern Brazil: a survival analysis. Matern Child Health 2012; 16(6):1257-65.

13. Lopes-Freire GM, Cárdenas ABC, Deza JEES, Ustrell-Torrent JM, Oliveira LB, Quesada Jr JRB. Exploring the association between feeding habits, non-nutritive sucking habits, and malocclusions in the deciduous dentition. Prog Orthod $2015 ; 16: 43$.

14. Germa A, Clément C, Weissenbach M, Heude B, Forhan A, Martin-Marchand L, et al. Early risk factors for posterior crossbite and anterior open bite in the primary dentition. Angle Orthod 2016; 86(5):832-8.

15. Warren JJ, Levy SM, Nowak AJ, Tang S. Non-nutritive sucking behaviors in preschool children: a longitudinal study. Pediatr Dent 2000; 22(3):187-91.

16. Çaglar E, Larsson E, Andersson EM, Hauge MS, Ogaard B, Bishara S, et al. Feeding, artificial sucking habits, and malocclusions in 3-year-old girls in different regions of the world. J Dent Child 2005; 72(1):25-30.

17. Katz CR, Rosenblatt A, Gondim PP. Nonnutritive sucking habits in Brazilian children: effects on deciduous dentition and relationship with facial morphology. Am J Orthod Dentofacial Orthop 2004; 126(1):53-7.

18. Buccini G dos S, Benício MH, Venancio SI. Determinants of using pacifier and bottle feeding. Rev Saúde Pública 2014; 48(4):571-82.

19. Carvalho AC, Paiva SM, Viegas CM, Scarpelli AC, Ferreira FM, Pordeus IA. Impact of malocclusion on oral health-related quality of life among Brazilian preschool children: a population-based study. Braz Dent J 2013; 24(6):655-61.

20. Kramer PF, Feldens CA, Ferreira SH, Bervian J, Rodrigues PH, Peres MA. Exploring the impact of oral diseases and disorders on quality of life preschool children. Community Dent Oral Epidemiol 2013; 41(4):327-35. 
21. Moimaz SAS, Rocha NB, Garbin AJI, Saliba O. The relation between maternal breast feeding and non-nutritive sucking habits. Ciênc Saúde Coletiva 2011; 16(5):2477-84.

22. Victora CG, Behague DP, Barros FC, Olinto MT, Weiderpass E. Pacifier use and short breastfeeding duration: cause, consequence, or coincidence? Pediatrics 1997; 99(3):445-53.

23. Solis CEM, Rosado JFC, Rosado AJD. Malos hábitos orales en infantes de guarderías del IMSS. Rev Med IMSS 2001; 39(5):435-40.

24. Feldens CA, Martins RP, Maciel RR, Vargas-Ferreira F, Kramer PF. Factors Associated with the occurrence of distoclusion in the primary dentition: a hierarchical analysis. J Clin Pediatr Dent 2016; 40(1):88-93.

25. Brasil. Ministério da Saúde. Secretaria de Atenção à Saúde. Departamento de Ações Programáticas e Estratégicas: II Pesquisa de Prevalência de Aleitamento Materno nas Capitais Brasileiras e Distrito Federal. Brasília: Ministério da Saúde; 2009.

26. Victora CG, Bahl R, Barros AJ, França GV, Horton S, Krasevec J, et al. Breastfeeding in the 21st century: epidemiology, mechanisms, and lifelong effect. Lancet 2016; 387(10017):475-90.

Endereço para correspondência:

Fabiana Vargas-Ferreira

Rua Expedicionário José Assumpção dos Anjos,

1130, Apto 102

31310-050, Belo Horizonte, Minas Gerais, Brasil

Telefone: (51) 996095029

E-mail: fabivfer@gmail.com

Recebido: 27/05/18. Aceito: 10/07/18. 